\title{
A1-3 chromosomal translocations in Italian populations of the peach potato aphid Myzus persicae (Sulzer) not linked to esterase-based insecticide resistance
}

\author{
Marco Rivi ${ }^{1}$, Valentina Monti ${ }^{1}$, Emanuele Mazzoni ${ }^{2}$, \\ Stefano Cassanelli ${ }^{1}$, Michela Panini ${ }^{2}$, Matteo Anaclerio ${ }^{2}$, \\ Manuela Cigolini ${ }^{2}$, Bruna Corradetti ${ }^{3}$, Davide Bizzaro ${ }^{3}$, \\ Mauro Mandrioli ${ }^{1}$ and Gian Carlo Manicardi ${ }^{1 *}$ \\ ${ }^{1}$ Dipartimento di Scienze della Vita, Università di Modena e Reggio Emilia, \\ Reggio Emilia, Italy: ${ }^{2}$ Istituto di Entomologia e Patologia vegetale, Università \\ Cattolica del Sacro Cuore, Piacenza, Italy: ${ }^{3}$ Dipartimento di Scienze della Vita \\ e dell' Ambiente, Università Politecnica delle Marche, Ancona, Italy
}

\begin{abstract}
Esterase-based resistance in the peach-potato aphid, Myzus persicae (Sulzer), is generally due to one of two alternative amplified carboxylesterase genes, E4 or FE4 (fast E4). The E4 amplified form is distributed worldwide and it is correlated with a particular translocation between autosomes 1 and 3, whereas the FE4 form, which has hitherto not been found to be associated with chromosomal rearrangements, is typical of the Mediterranean regions. In this study, we present for the first time cytogenetic and molecular data on some M. persicae parthenogenetic lineages, which clearly show a chromosomal A1-3 translocation associated with esterase FE4 genes and unrelated to high levels of esterase-based resistance.
\end{abstract}

Keywords: chromosomal rearrangements, esterase, gene amplification, insecticide resistance, E4 and FE4, Myzus persicae

(Accepted 11 September 2012; First published online 1 March 2013)

\section{Introduction}

Esterase-based resistance to organophosphorous and carbamate insecticides is common in different insect pests (Bass \& Field, 2011). They produce either a broad-spectrum insecticide resistance through rapid-binding and slow turnover of insecticide, i.e., sequestration, or a narrow spectrum insecticide resistance through metabolism of a very restricted range of insecticides containing a common ester bond (Karunaratne et al., 1995). The majority of esterases

*Author for correspondence

Phone: +39-0522-522059; Fax: +39-0522-522027

E-mail: giancarlo.manicardi@unimore.it functioning by sequestration are elevated through gene amplification (Bass \& Field, 2011).

In the peach potato aphid Myzus persicae (Sulzer) (Hemiptera: Aphididae), there are two common esterase variants, E4 and FE4 (fast E4), which appear to result from independent amplification events. Detailed molecular studies have established that E4 and FE4 genes are part of a gene family since they differ by only nine amino-acid substitutions (Field et al., 1993; Field \& Devonshire, 1998). The corresponding enzymes differ slightly in their molecular mass, 65 and $66 \mathrm{kDa}$ for E4 and FE4, respectively, as a consequence of a nonsense mutation bringing to a premature stop codon in the E4 gene (Devonshire et al., 1998). Despite their very similar molecular weights, FE4 isoform appears slightly faster when run on electrophoresis gels (Devonshire, 1989). Both gene forms have seven introns within the coding region which are 
of the same size and in identical positions showing $97 \%$ sequence identity (Field et al., 1996).

The amount of esterase is variable among populations, and in early studies a relationship between resistance level and enzymatic activity has been found (Needham \& Sawicki, 1971). In extremely resistant $\left(R_{3}\right)$ strains, these enzymes can constitute up to $1 \%$ of the total aphid protein (Devonshire et al., 1998). In situ hybridization studies have demonstrated that in susceptible aphids, E4 and FE4 genes occur as a single copy, E4 being localized $19 \mathrm{~kb}$ upstream to FE4 (Field \& Devonshire, 1998). Current literature data report that resistant aphids have one of two alternative amplified carboxylesterase genes, E4 or FE4, according to their karyotype. The E4 amplified genes occur at a single chromosomal location, on autosome $3^{\mathrm{T}}$ closely linked with an A1-3 chromosomal translocation, whereas the FE4 genes have multiple sites of insertion, which are apparently not associated with chromosomal rearrangements (Blackman et al., 1999). Translocated E4-producing aphids are highly widespread in warm and tropical regions and in glasshouse in the Northern Europe. Mild winters or lack of the primary host (the peach Prunus persica) force such populations to reproduce all the year around by parthenogenesis on one or more secondary herbaceous hosts thus avoiding the bisexual phase of their life cycle involving host alternation governed by both ambient temperature and prevailing day length conditions (Blackman \& Eastop, 2000). Conversely, FE4-based resistance has developed in peach-growing areas in Southern Europe, where it is associated with the normal karyotype $(2 n=12)$ and an annual sexual phase (Blackman et al., 1995).

Studies investigating the presence and diffusion of insecticide resistance performed both by biochemical and molecular assays (Bizzaro et al., 2005; Criniti et al., 2008) reveal that many Italian populations of $M$. persicae possess the amplified FE4 gene only and highly variable levels of methylation, known to be associated with epigenetic control of esterase genes (Field \& Blackman, 2003).

In the present study, we performed cytogenetic and molecular assays on 38 Italian parthenogenetic lineages collected from primary (peach) and secondary hosts, showing for the first time $M$. persicae populations to have a chromosomal A1-3 translocation coupled with esterase FE4 genes, but unrelated to high levels of esterase-based resistance.

\section{Material and methods}

\section{Aphids}

M. persicae populations were collected not only from peach orchards (25) but also from herbaceous hosts including tobacco (nine), tomato (two), potato (one) and aubergine (one) in different Italian localities (table 1). In addition, a fully susceptible strain (US1L) kindly provided by Dr Alan Devonshire (Rothamsted Research, Harpeden, UK) was used as a reference. All strains were maintained as parthenogenetic lineages starting from a single parthenogenetic female on pea-seedlings (Pisum sativum cv 'Meraviglia d'Italia') under constant ambient conditions viz: $21 \pm 0.5^{\circ} \mathrm{C}$ and a long-day photoperiod of $16 \mathrm{~h}: 8 \mathrm{~h}$ light:dark.

\section{Chromosome preparation}

Chromosome preparations of parthenogenetic females were obtained by spreading embryo cells, as previously described by Bizzaro et al. (1996).

\section{Biochemical assays}

Total esterase activity was biochemically assayed in 96well microplates in a Tecan Genios pro reader using $\alpha$ naphthylacetate as a substrate as previously described (Mazzoni \& Cravedi, 2002; Bizzaro et al., 2005). Absorbance values were converted to $\alpha$-naphthol per protein unit (nmoles $\mathrm{mg}^{-1}$ ) using an $\alpha$-naphthol standard curve. Total protein content was measured in microplates using a 'Biorad Protein assay kit' according to manufacturer instructions. Each population was assayed at least in triplicate.

\section{DNA extraction}

Genomic DNA (gDNA) was extracted from pools of 30 parthenogenetic females through a standard Sodium Dodecyl Sulphate SDS/proteinase K/Phenol-Chloroform protocol (Bizzaro et al., 2005).

gDNA was quantified using a Nanodrop spectrophotometer and its quality checked through $1 \%$ agarose gel electrophoresis in $0.5 \times \mathrm{TBE}$ buffer.

\section{Detection of the amplified E4/FE4 gene sets and evaluation of single site methylation level}

Two PCR-based strategies were employed to establish whether FE4 and/or E4 esterase genes were amplified in the examined strains. Following the method of Field et al. (1999) and Field \& Foster (2002), the 5' co-amplification of both FE4 and E4 genes produces $865 \mathrm{bp}$ and $572 \mathrm{bp}$ fragments in FE4 and E4 amplified aphid's genome, respectively, while only faint bands were visible in insects displaying the wild-type genome after agarose gel electrophoresis. The amplified genes encoding carboxylesterases FE4 and E4 were also confirmed according to a polymerase chain reaction-restriction fragment length polymorphism (PCRRFLP) early developed by Field et al. (1996) and later modified by Guillemaud et al. (2003). This technique allows the amplification of the region spanning the intron 7 using the couple of primers: F (5'-AAATCATATTTCCCGGGTTC) R (5'AGGTTCACTAAGATTACTCA) shared between E4 and FE4 gene sequences. The products so obtained was cut by single site restriction enzymes HindIII (specific for FE4 gene) and SpeI (specific for E4 gene), and separated on agarose gel electrophoresis. The PCR product obtained from the reference strain and strains bearing the A1-3 chromosomal translocation were also similarly cut and sequenced. As described by Field et al. (1996), since the 3' of the forward primer used for the last PCR products occurs at a methylation-sensitive HpaII site, the successful amplification after HpaII digestion of the gDNA, reveals the methylation pattern of the amplified genes at that site.

\section{Determination of gene copy ratio by quantitative real-time PCR ( $q P C R)$}

Quantification of the relative E4/FE4 copy number between the Italian and the reference strains was performed according to the comparative $2^{-\Delta \Delta C t}$ method (Livak \& Schmittgen, 2001; Pfaffl, 2001). Primer sequences employed for the qPCR of target genes E4/FE4 (Genbank accession nos. X74554.1 and X74555.1) and for the reference gene, $\beta$-actin gene, have been previously described by Kwon et al. 
Table 1. Total esterase activity ( $\alpha$-naphthol/protein; nmoles $\mathrm{mg}^{-1}$ ), gene copy ratio and chromosome number in Italian populations of $M$. persicae. 12t indicates the presence of the A1-3 chromosomal translocation. Frm followed by X or a number indicates the occurrence of a specific chromosomal fragmentation.

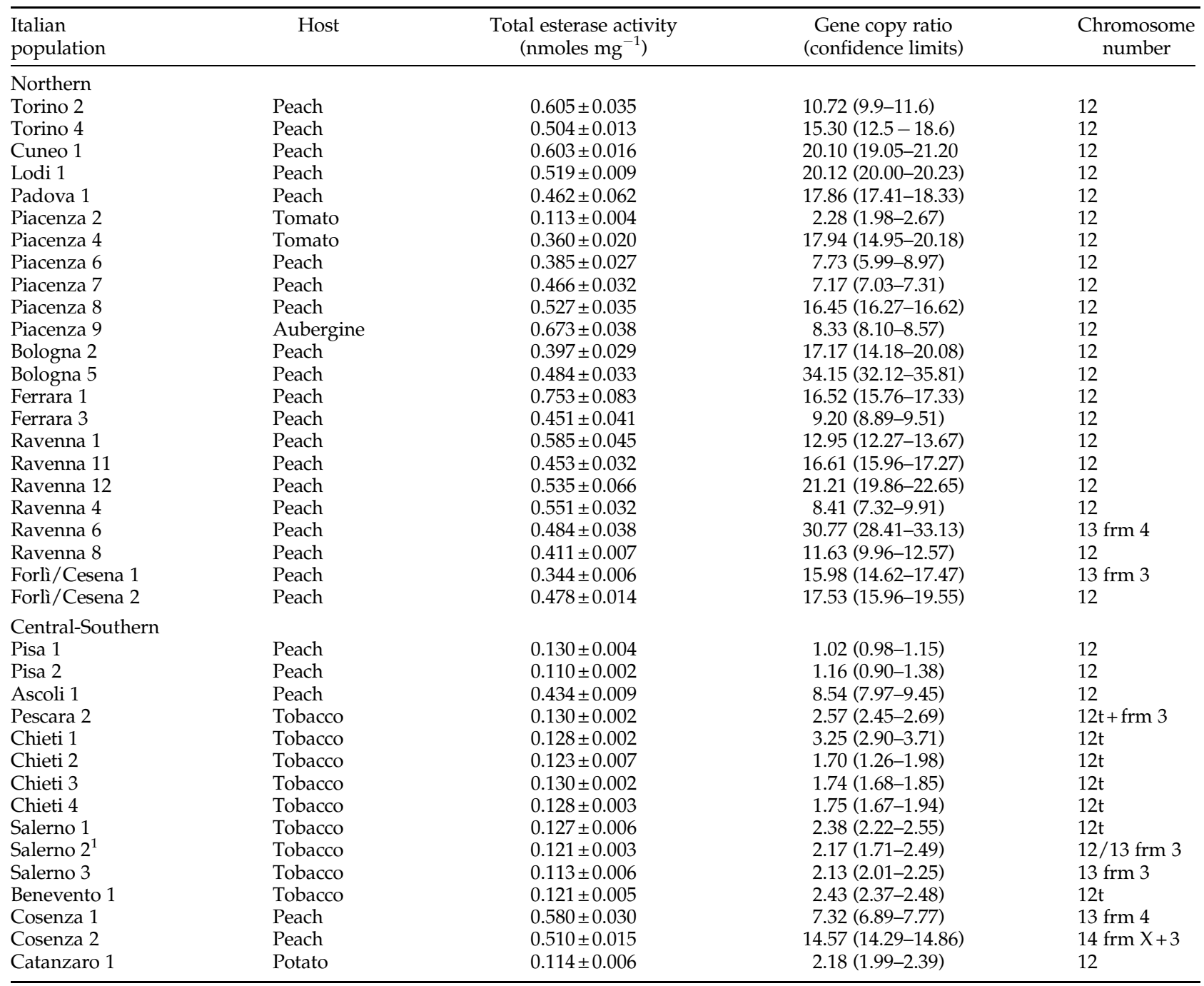

${ }^{1}$ Salerno 2 is a case of intra-individual chromosomal mosaicism.

(2009). The amplification efficiency of qPCR was adjusted close to $\sim 2.0$ for both genes using the following equation: $E=10^{-1}$ per slope. The slope was derived from titration curves consisting in five serial dilutions (6$0.25 \mathrm{ng} \mu \mathrm{l}^{-1}$ ) of gDNA from reference strain (four independent extractions) with three technical replications for each point (resulting slopes $-3.35 \pm 1$ and $-3.37 \pm 1$ for E4/FE4 and $\beta$ actin standard curves, respectively). The optimized qPCR mix $(25 \mu \mathrm{l}$ as a final volume) contained $1 \times$ SYBR Green master mix (Promega), $5 \mu \mathrm{l}$ DNA (equivalent to $5 \mathrm{ng}$ of total gDNA) and $5 \mu$ gene specific primes at a final concentration of $5 \mu \mathrm{M}$. A 'notemplate' negative control and all samples were analyzed in triplicate. The reaction cycle performed in an ABI 7300 Real Time PCR system (Applied Biosystems), comprised a melting (denaturation) step of $95^{\circ} \mathrm{C}$ for $2 \mathrm{~min}$, followed by 40 cycles of $94^{\circ} \mathrm{C}$ for $10 \mathrm{~s}$, annealing at $56^{\circ} \mathrm{C}$ for $10 \mathrm{~s}$ and an extension step at $72^{\circ} \mathrm{C}$ for $15 \mathrm{~s}$. Specificity of the PCRs was also assessed by a melting curve analysis for each PCR by examination of the dissociation curve so produced. The qPCR experiments were repeated three times with independently extracted total DNA for each strain.

\section{Statistical analysis}

Correlation between gene copy ratio and total esterase activity was evaluated using the CURVEFIT procedure of SPSS Statistics (version 19).

\section{Results}

\section{Cytogenetic analysis}

A cytogenetic overview performed on mitotic metaphases obtained after spreading $M$. persicae embryo cells, revealed 


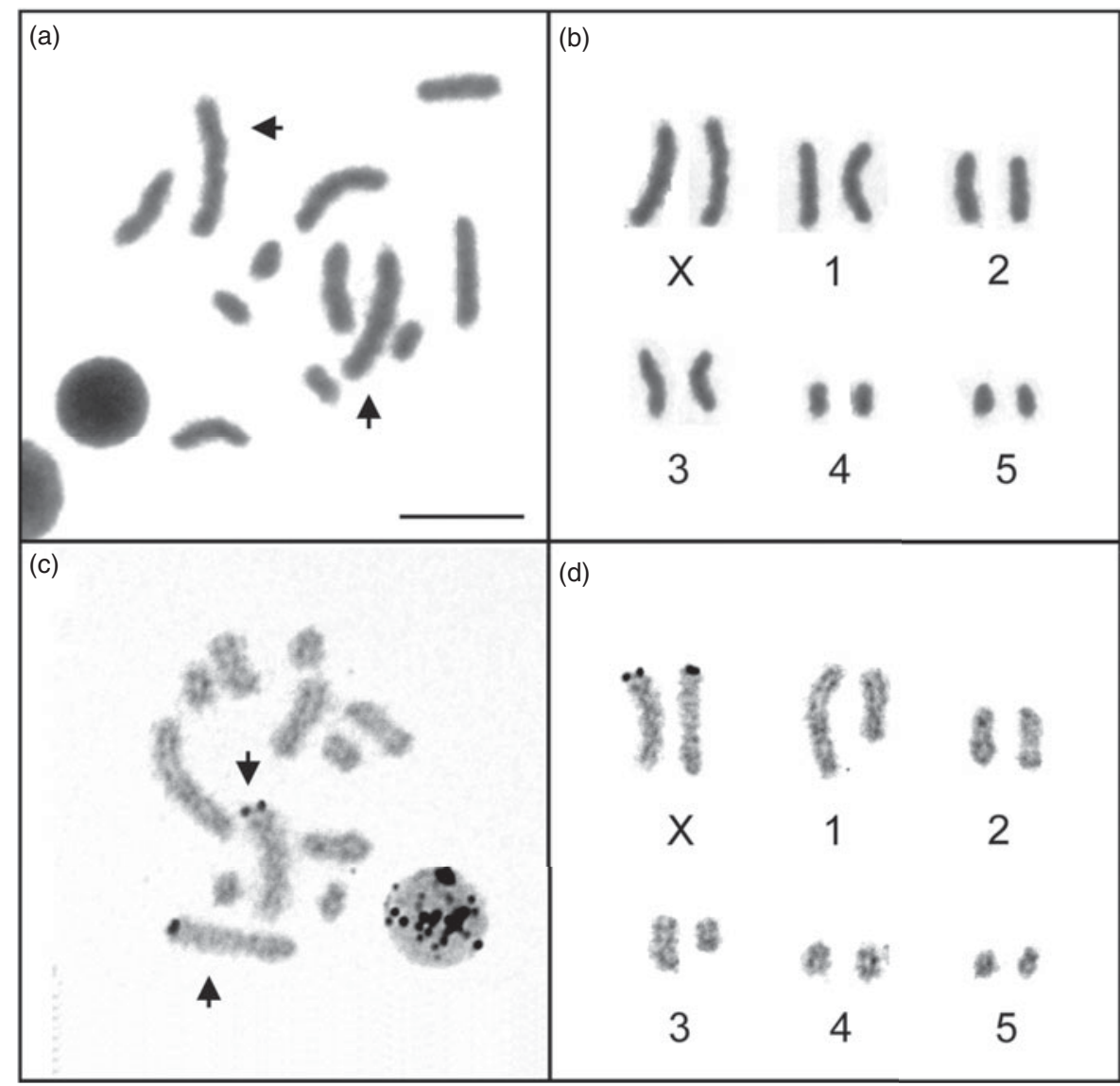

Fig. 1. Giemsa staining of chromosomes of two different $M$. persicae populations: $2 n=12$ chromosome complement (a) and the corresponding karyotype (b); metaphase with A1-3 chromosomal translocation (c) and the corresponding karyotype (d). Bar corresponds to $10 \mu \mathrm{m}$.

that 13 of the 38 strains analyzed possess karyotypic variants. Among these, the more frequent was an autosomal A1-3 chromosomal translocation (table 1) (fig. 1). Table 1 clearly shows that all the strains with this particular translocation had very low esterase activity levels and that their FE4 gene copy ratios varied between 1.7 and 3.2 when compared with the reference strain.

\section{Biochemical assays}

Most of the populations collected in Northern Italy show a total esterase activity comparable with that of resistant strains, whereas populations with low total esterase activity are largely confined to the Southern-Central region of the country (table 1).

\section{Identification of E4 and/or FE4 genes}

In order to gain a clear-cut distinction between E4 and FE4 gene amplification, we compared the PCR assays with those published in the literature (Field et al., 1996, 1999). We observed a single electrophoretic band corresponding to the $865 \mathrm{bp}$ region, diagnostic for the FE4 gene, in all $M$. persicae strains analyzed using primers amplifying the 5'-end of the esterase gene. Similarly, the PCR-RFLP assay showed that only the restriction enzyme HindIII was able to digest the amplified fragment thus confirming that FE4 is the only esterase gene amplified in all the analyzed Italian populations. Successful PCR amplification of a $670 \mathrm{bp}$ FE4 esterase fragment, even after digestion with the methylation sensitive HpaII restriction enzyme located within one of the PCR primer pairs, indicates that all the FE4 esterase genes are methylated at this position.

\section{Analysis of PCR sequences obtained using primers EST $3 N-E S T 4 P$ on gDNAs of different $\mathrm{M}$. persicae strains}

In order to provide further information on the esterase gene(s) in Italian samples of $M$. persicae, we fully sequenced the PCR product obtained after amplification of gDNA of the reference strain and three strains possessing the A1-3 chromosomal translocation (Chieti 2, Chieti 4 and Salerno 1) with the primers EST 3N-EST 4p. Sequences analysis confirmed that, in the translocated strains, these particular primers amplify the FE4 genes exclusively (fig. 2).

\section{Determination of gene copy number}

Real-time PCR estimation of the esterase copy ratio (obtained by comparing the value of the reference strain 
primer EST $3 \mathbf{N}$

Ref. strain AAATCATATTTCCCGGGTTCAAGTAAATAATATATTTTAAATACCTACTTTTGTATGTGTAT

Chieti 2

Chieti 4

Salerno 1

Ref. strain

Chieti 2

Chieti 4

Salerno 1 AATACCTACTTTTGTATGTGTAT -AATAACTACTTTTGTATGTGTAT -AATACCTACTTTTGTATGTGTAT

Ref. strain AGCTATGTATTAAAAATGGATGGTTTCTACGTTTACGACAATGAAGAAGATAGAAAGATGA Chieti 2 AGCTATGTATTAAAAATGGATGGTTTCAACGTTTACGACAATGAAGAAGATAGAAAGATGA Chieti 4 agCTATGTATTAAAAATGGATGGTTTCACGTTTACGACAATGAAGAAGATAGAAAGATGA Salerno 1 AGCTATGTATTAAAAATGGATGGTTTCTACGTTTACGACAATGAAGAAGATAGAAAGATGA

Ref. strain TCAAAACTATGGTTAATATTTGGGCAACTTTTATTAAATCTGGGTAATTATCTGACTTTTA Chieti 2 TCAAAACTATGGTTAATATTTGCGCAACTTTTATTAAATCTGGGTAATTATCTGACTTTTA Chieti 4 TCAAAACTATGGTTAATATTTGGGCAACTTTTATTAAATCTGGGTAATTATCTGACTTTTA Salerno 1 TCAAAACTATGGTTAATATTTGCGCAACTTTTATTAAATCTGGGTAATTATCTGACTTTTA

Ref. strain TTGTTTGATACTCTAATCAATCATTGTAATGAGTATTAATGGAATCTGATATTAGTACCTA Chieti 2 TtATTTGATACTCTACTCAATCATTGTAATGAGTATTAATGGAATCTGATATTAGTACCTA Chieti 4 TTATTTGACACTCTAATCAATCATTGTAATGAGTATTAATGGAATCTGATAATAGTACCTA Salerno 1 TTATTTGATACTCTAATCAATCATTGTAATGAGTATTAATGGAATCTGATATTAGTACCTA

Ref. strain AACTGATTCTAGCCTAAACTCCATAGTCTGTTCAGAATTAAATCAGCACAGGCTGCCACCT Chieti 2 AaCAGATTCTAGCGTAAACTCCATAGTCTGTTCAGAATTAAATCAGCACAGGCTGCCACCT Chieti 4 AACTGATTCTAGCCTAAACTCCATAGTCTGTTCATAATTAAATCACCTCAGGCTGCCACCT Salerno 1 AACTGATTCTAGCCTAAACTCCATAGTCTGTTCAGAATTAAATCAGCACAGGCTGCCACCT

Ref. strain ACTGAGTATAGGTGTGGCTTTGTTTCCTGTACCTACCGTACTCATTTTTAACAGACTATAA Chieti 2 ACTGAGTATAGGTGTGGCTTTGTTTCCTGTACCTACCGTACTCATTTTTAACAGACTATAA Chieti 4 ACTGAATATATGTGTGGCTTTGTTTCCTGTACCTACCTTACTCATTTTTAACAGACTATAA Salerno 1 ACTGAGTATAGGTGTGGCTTTGTTTCCTGTACCTACCGTACTCATTTTTAACAGACTATAA

Fig. 2. See opposite page for legend. 


\section{HindIII}

Ref. strain TTAATACATATTAAAAAAATAAAGCTTTCAAATCTTAAACAATAAATATTTTATATTTTTT

Chieti 2 TTAATACATATTAAAAAAATAAAGCTTTCAAATCATAAACAATAAATATTTTATATTTTTT

Chieti 4 TTAATACATATTAAAAAAATAAAGCTTTCAAATCATAAACAATAAATATTTTATATTTTTT

Salerno 1 TTAATACATATTAAAAAAATAAAGCTTTCAAATCTTAAACAATAAATATTTTATATTTTTT

SpeI

Ref. strain TATACATTCTAGGCTGTATTAACTAGTGATTATAATTTTTTTTTTTTTTACTTTATAGAGT

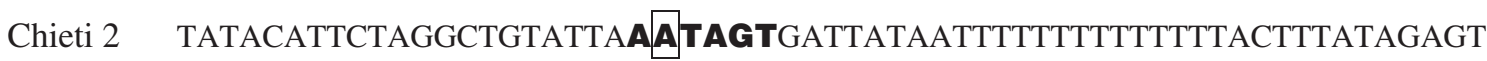

Chieti 4 TATACATTCTAGGCTGTATTAAATAGTGATTATAATTTTTTTTTTTTTTACTTTATAGAGT

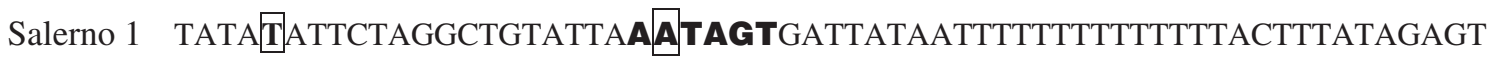

Ref. strain ACCAGATACTGAAAATTCAGAAATTTGGTTACCTGTTTCTAAGAATCCAGCAGATCCTTLC

Chieti 2 ACCAGATACTGAAAATTCAGAAATTTGGTTACCTGT-

Chieti 4 ACCAGATACTGAAAATTCAGAAATTCGGTTACCTGT-

Salerno 1 ACCAGATACTGAAAATTCAGAAATTTGGTTACCTGT-

primer EST 4p

Ref. strain AGGTTCACTAAGATTACTCA

Chieti 2

Chieti 4

Salerno 1

Fig. 2. PCR products sequences obtained by amplifying gDNA extracted from three A1-3 translocated strains (Chieti 2, Chieti 4 and Salerno 1) and from the reference strain using EST 3N-EST $4 \mathrm{p}$ primers. HindIII and SpeI restriction sites are evidenced in bold. The nucleotides marked with square are specific for the FE4 esterase form. Simple nucleotides polymorphisms are marked in grey. Primer sequences used for both amplification and sequencing are evidenced in underlined characters.

with that of the different Italian populations) showed that the analyzed populations possessed a gene copy ratio ranging from 1 to 34 (table 1). Statistical analysis estimated a high positive logarithmic regression coefficient between gene copy ratio and total esterase activity $\left(r^{2}=0.71 ; P<0.01 ; n=38\right)$ (fig. 3).

\section{Discussion}

During a population screening of Italian peach potato aphids, M. persicae for karyotype variants, we characterized, for the first time in Italy, strains showing an autosomal A1-3 chromosomal translocation (Rivi et al., 2009, Rivi et al., 2012).

Up until this time, such a chromosomal rearrangement has been worldwide associated with the conferment of insecticide resistance involving amplification of the esterase E4 gene (Blackman, 1971; Blackman \& Devonshire, 1978; Takada, 1986; Blackman et al., 1995), with the only exception of some Japanese populations, for which no clear association

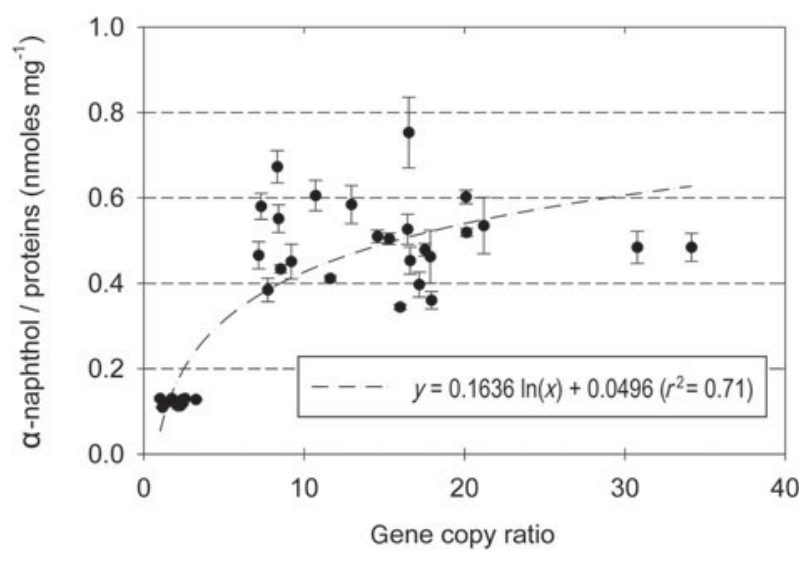

Fig. 3. Relationship between gene copy ratio and total esterase activity, reported as $\alpha$-naphthol/protein $\left(\right.$ nmoles $\left.\mathrm{mg}^{-1}\right)( \pm \mathrm{SE})$, in each strain of $M$. persicae studied. 
between resistance level and karyotype was found (Takada, 1979). No data were previously available for the situation pertaining to Italy. In order to fill this gap, we combined two molecular screening methods (Field et al., 1996, 1999) together with sequence analysis highlighting that, in all the Italian strains examined, insecticide resistance is associated only with the FE4 gene. Moreover, biochemical and molecular analyses showed that all A1-3 translocated strains possess very low esterase activity and an esterase gene copy ratio varying between 1.7 and 3.2. The aforementioned data set is contrary to previous finding as published in the literature in which the translocaiton is always associated with enhancement of the E4 geene and clearly reveals that, at least in Italian FE4-bearing strains, the origin of this particular chromosomal rearrangement is unrelated with esterase based resistance. In light of this, we hypothesize that the observed chromosomal rearrangement depends on a fragile site located on autosome 3. Due to the holocentric nature of aphid chromosomes, this fragment is stable enough to be maintained and inherited in following generations, even those following sexual reproduction in the autumn. $M$. persicae strains showing a karyotype $2 \mathrm{n}=13$ with $\mathrm{A} 3$ fragmentation have been described in different parts of the world (Blackman, 1980), including the two populations analysed in this paper. This fragment frequently translocates on one telomere of autosome 1 which is evidently more prone to accept this fragment compared with fragments from the other chromosomes. FISH (fluoresecent in situ hybridization) experiments suggest that, both in E4 and unamplified FE4 wild type strains, esterase genes map close to a subtelomeric block of heterochromatic repetitive DNA on autosome 1, within the region in which translocation occurs (Blackman et al., 1999). Thus, the translocation process could be responsible for the activation of the molecular mechanisms involved in the E4 gene amplification as earlier suggested by Blackman et al. (1978). The worldwide diffusion of this rearrangement might be favoured by (i) apomictic parthenogenesis typical of aphids; (ii) anholocycly which allows individuals to escape gene rearrangement which would otherwise occur during the autumnal sexual reproducing phase; (iii) insecticide applications as the selection factor primarily responsible for the amplification of esterase genes, and which reduces the efficacy of organophosphate, carbamate as well as pyrethroid insecticides (Ffrench-Constant et al., 1987; Devonshire et al., 1998; Foster et al., 2000).

To explain the strong correlation between gene amplification and A1-3 translocation limitedly to strains possessing E4 form, it is of interest that amplified esterase genes are located only at a single heterozygous site $3^{\mathrm{T}}$ in $\mathrm{E} 4$ strains, having been moved there from autosome 1 in the course of a reciprocal exchange (Field \& Blackman, 2003). Conversely, in FE4 strains, esterase genes have multiple locations frequently involving different chromosomes (Field \& Blackman, 2003) so that specimens with normal karyotype $(2 n=12)$ could survive insecticide treatments as a consequence of esterase gene amplification at other loci.

In addition to A1-3 translocation, we describe more uncommon chromosomal rearrangements (table 1). The small number of observations prevents any correlation being made between these karyotype variations and the esterasebased resistance, but, noteworthy, is the fact that both the strains showing A4 fragmentations have a high esterase-based activity. Even so, further investigations are required to confirm this apparent association.
This overview, regarding the molecular basis of esterase insecticide resistance in Italian populations of $M$. persicae, is in accordance with previous data attesting that, in Italy, esterase insecticide resistance is based on FE4 amplification and, at least in the recent past, has proved a serious problem for aphid management since most of the analyzed populations showed a total esterase activity comparable with resistant or highly resistant strains (Bizzaro et al., 2005; Criniti et al., 2008). Moreover, as already reported for strains with E4 (Field et al., 1999) or FE4 gene amplification (Bizzaro et al., 2005), statistical analysis performed on Italian strains reveals the existence of a positive relationship between the FE4 gene copy ratio and esterase activity, in turn indicating that gene amplification is the main cause of carboxylesterase overproduction in M. persicae in place of the FE4 over-expression.

\section{References}

Bass, C. \& Field, L.M. (2011) Gene amplification and insecticide resistance. Pest Management Science 67, 886-890.

Bizzaro, D., Manicardi, G.C. \& Bianchi, U. (1996) Chromosomal localization of a highly repeated EcoRI DNA fragment in Megoura viciae (Homoptera, Aphididae) by nick translation and fluorescence in situ hybridization. Chromosome Research 4, 392-396.

Bizzaro, D., Mazzoni, E., Barbolini, E., Giannini, S., Cassanelli, S., Pavesi, F., Cravedi, P. \& Manicardi, G.C. (2005) Relationship among expression, amplification, and methylation of FE4 esterase genes in Italian populations of Myzus persicae (Sulzer) (Homoptera: Aphididae). Pesticide Biochemistry and Physiology 81, 51-58.

Blackman, R.L. (1971) Chromosomal abnormalities in an anholocyclic biotype of Myzus persicae (Sulzer). Experientia 27, 704-706.

Blackman, R.L. \& Devonshire, A.L. (1978) Further studies on the genetics of the carboxylesterase regulatory system involved in resistance to organophosphorus insecticides in Myzus persicae (Sulzer). Pesticide Science 9, 517-521.

Blackman, R.L. \& Eastop, V.F. (2000) Aphids of the Worlds' Crops. 2nd edn. John Wiley \& Sons Ltd. Chicester, U.K.

Blackman, R.L., Takada, H. \& Kawakami, K. (1978) Chromosomal rearrangement involved in insecticide resistance of Myzus persicae. Nature 271, 450-452.

Blackman, R.L., Spence, J.M., Field, L.M. \& Devonshire, A.L. (1995) Chromosomal location of the amplified esterase genes conferring resistance to insecticides in Myzus persicae (Homoptera: Aphididae). Heredity 75, 297-302.

Blackman, R.L., Spence, J.M., Field, L.M. \& Devonshire, A.L. (1999) Variation in the chromosomal distribution of amplified esterase (FE4) genes in Greek field populations of Myzus persicae (Sulzer). Heredity 82, 180-186.

Criniti, A., Mazzoni, E., Cassanelli, S., Cravedi, P., Tondelli, A., Bizzaro, D. \& Manicardi, G.C. (2008) Biochemical and molecular diagnosis of insecticide resistance conferred by esterase, MACE, $k d r$ and super- $k d r$ based mechanisms in Italian strains of the peach potato aphid, Myzus persicae (Sulzer). Pesticide Biochemistry and Physiology 90, 168-174.

Devonshire, A.L. (1989) The role of electrophoresis in the biochemical detection of insecticide resistance. pp. 363-374 in Loxdale, H.D. \& den Hollander, J. (Eds) Electrophoretic Studies on Agricultural Pests. Clarendon Press, Oxford.

Devonshire, A.L., Field, L.M., Foster, S.P., Moores, G.D., Williamson, M.S. \& Blackman, R.L. (1998) The evolution of insecticide resistance in the peach-potato aphid, Myzus 
persicae. Philosophical Transactions of the Royal Society of London. Series B, Biological Sciences 353, 1677-1684.

Ffrench-Constant, R.H., Devonshire, A.L. \& Clark, S.J. (1987) Differential rate of selection for resistance by carbamate, organophosphorus and combined pyrethroid and organophosphorus insecticides in Myzus persicae (Sulzer) (Hemiptera: Aphididae). Bulletin of Entomological Research 77, 227-238.

Field, L.M. \& Blackman, R.L. (2003) Insecticide resistance in the aphid Myzus persicae (Sulzer): chromosome location and epigenetic effects on esterase gene expression in clonal lineages. (Intraclonal genetic variation: ecological and evolutionary aspects.) Biological Journal of the Linnean Society 79, 107-113.

Field, L.M. \& Devonshire, A.L. (1998) Evidence that the E4 and FE4 esterase genes responsible for insecticide resistance in the aphid Myzus persicae (Sulzer) are part of a gene family. Biochemical Journal (London) 330, 169-173.

Field, L.M. \& Foster, S.P. (2002) Amplified esterase genes and their relationship with other insecticide resistance mechanisms in English field populations of the aphid, Myzus persicae (Sulzer). Pest Management Science 58, 889-894.

Field, L.M., Williamson, M.S., Moores, G.D. \& Devonshire, A.L. (1993) Cloning and analysis of the esterase genes conferring insecticide resistance in the potato-peach aphid, Myzus persicae. Biochemical Journal (London) 294, 569-574.

Field, L.M., Crick, S.E. \& Devonshire, A.L. (1996) Polymerase chain reaction-based identification of insecticide resistance genes and DNA methylation in the aphid Myzus persicae (Sulzer). Insect Molecular Biology 5, 197-202.

Field, L.M., Blackman, R.L., TylerSmith, C. \& Devonshire, A.L. (1999) Relationship between amount of esterase and gene copy number in insecticide-resistant Myzus persicae (Sulzer). Biochemical Journal (London) 339, 737-742.

Foster, S.P., Denholm, I. \& Devonshire, A.L. (2000) The ups and downs of insecticide resistance in peach-potato aphids (Myzus persicae) in the UK. Crop Protection 19, 873-879.

Guillemaud, T., Brun, A., Anthony, N., Sauge, M.H., Boll, R., Delorme, R., Fournier, D., Lapchin, L. \& Vanlerberghe Masutti, F. (2003) Incidence of insecticide resistance alleles in sexually-reproducing populations of the peach-potato aphid Myzus persicae (Hemiptera: Aphididae) from southern France. Bulletin of Entomological Research 93, 289-297.

Karunaratne, S.H.P.P., Hemingway, J., Jayawardena, K.G.I., Dassanayaka, V. \& Vaughan, A. (1995) Kinetic and molecular differences in the amplified and non-amplified esterases from insecticide-resistant and susceptible Culex quinquefasciatus mosquitoes. Journal of Biological Chemistry 270, 31124-31128.

Kwon, D., Choi, B., Lee, S., Clark, J.M. \& Lee, S. (2009) Characterization of carboxylesterase-mediated pirimicarb resistance in Myzus persicae. Pesticide Biochemistry and Physiology 93, 120-126.

Livak, K.J. \& Schmittgen, T.D. (2001) Analysis of relative gene expression data using real-time quantitative PCR and the 2 (-Delta Delta C(T)) Method. Methods (San Diego, California) 25, 402-408.

Mazzoni, E. \& Cravedi, P. (2002) Analysis of insecticideresistant Myzus persicae (Sulzer) populations collected in Italian peach orchards. Pest Management Science 58, 975-980.

Needham, P.H. \& Sawicki, R.M. (1971) Diagnosis of resistance to organophosphorus insecticides in Myzus persicae (Sulz.). Nature 230, 125-126.

Pfaffl, M.W. (2001) A new mathematical model for relative quantification in real-time RT-PCR. Nucleic Acids Research 29, e45.

Rivi, M., Mazzoni, E., Criniti, A., Cassanelli, S., Bizzaro, D. \& Manicardi, G.C. (2009) Relationship between chromosomal translocation and FE4 gene amplification in an Italian population of the peach-potato aphid Myzus persicae (Hemiptera: Aphididae). Redia 92, 229-231.

Rivi, M., Monti, V., Mazzoni, E., Cassanelli, S., Panini, M., Bizzaro, D., Mandrioli, M. \& Manicardi, G.C. (2012) Karyotype variations in Italian populations of the peach potato aphid Myzus persicae (Hemiptera: Aphididae). Bulletin of Entomological Research 102, 663-671.

Takada, H. (1979) Estersae variation in Japanese populations of Myzus persicae (Sulzer) (Homoptera: Aphididae), with special reference to resistance to organophosphorous insecticides. Applied Entomology and Zoology 14, 245-255.

Takada, H. (1986) Genotypic composition and insecticide resistance of Japanese populations of Myzus persicae (Sulzer) (Hom., Aphididae). Journal of Applied Entomology 102, 19-38. 